Document downloaded from:

http://hdl.handle.net/10251/105893

This paper must be cited as:

Giner-Sanz, JJ.; Ortega Navarro, EM.; Pérez-Herranz, V. (2017). Harmonic Analysis Based Method for Perturbation Amplitude Optimization for EIS Measurements. Journal of The Electrochemical Society. 164(13):H918-H924. doi:10.1149/2.1451713jes

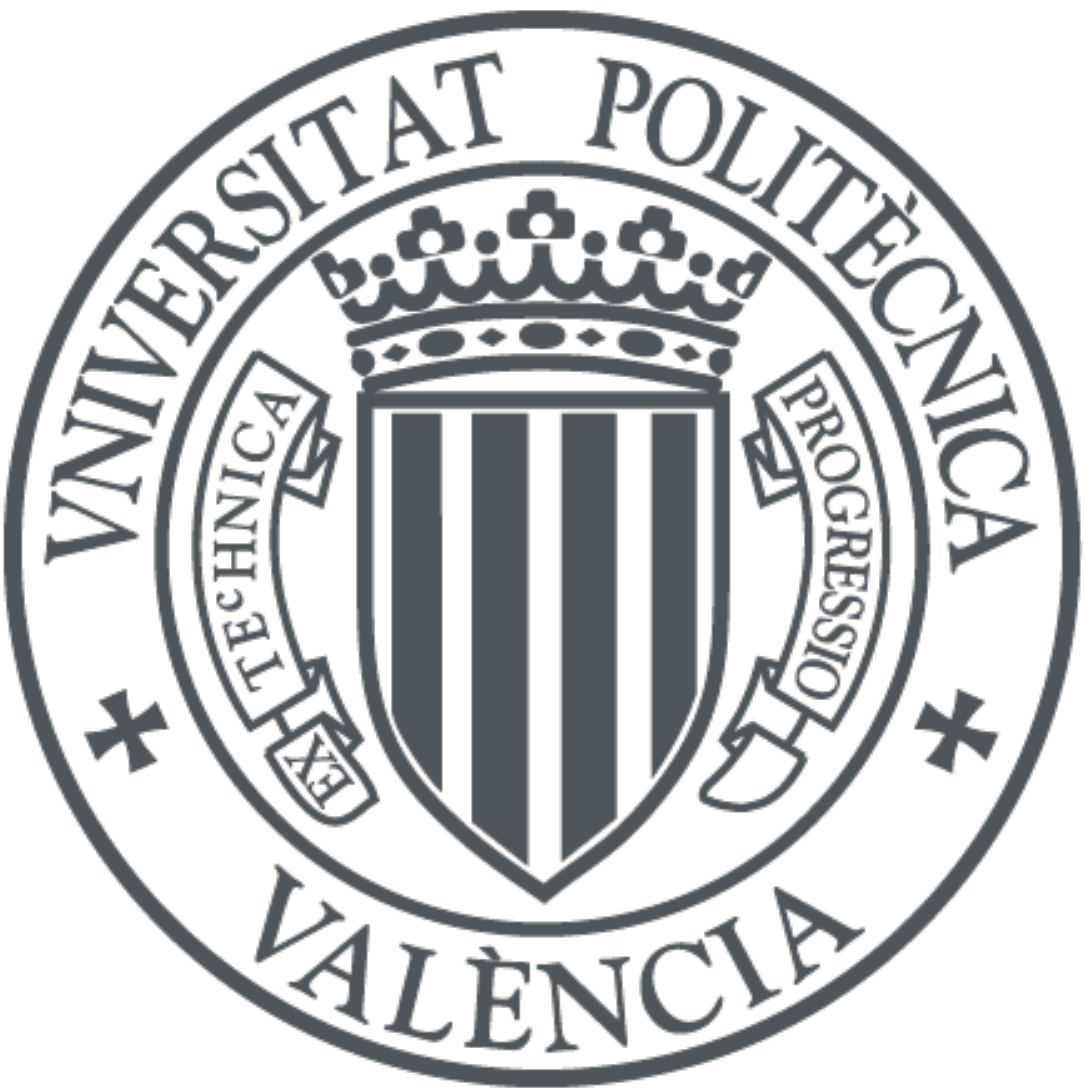

The final publication is available at

http://dx.doi.org/10.1149/2.1451713jes

Copyright The Electrochemical Society

Additional Information 


\title{
Harmonic analysis based method for perturbation amplitude optimization for EIS measurements
}

\author{
J. J. Giner-Sanz, E. M. Ortega, V. Pérez-Herranz* \\ IEC group, Depto. Ingeniería Química y Nuclear, Universitat Politècnica de València \\ Camino de Vera S/N, 46022 Valencia, Spain \\ *Corresponding author. Tel.: +34-96-3877632; fax: +34-96-3877639; \\ E-mail address: vperez@iqn.upv.es (V.Pérez-Herranz)
}

\begin{abstract}
$\underline{\text { Abstract }}$
The impedance concept is defined by Ohm's generalized law. Ohm's law requires the fulfilment of 3 conditions in order to be valid: causality, linearity and stability. In general, electrochemical systems are highly nonlinear systems; and therefore, in order to achieve linearity low amplitude perturbations have to be used during EIS measurements. However, small amplitude perturbations lead to low signal-to-noise ratios. Consequently, the quality of an EIS measurement is determined by a trade-off: the perturbation amplitude should be big enough in order to obtain a good signal-to-noise ratio; and at the same time, it should be small enough in order to avoid significant nonlinear effects. The optimum perturbation amplitude corresponds with the maximum perturbation amplitude that ensures a pseudo linear response of the system. In this work, a method for experimentally determining the optimum perturbation amplitude for performing EIS measurements of a given system is presented. The presented method is based on the harmonic analysis of the output signals; and in this work, it was applied to a highly nonlinear system: the cathodic electrode of an alkaline water electrolyser. The presented method allows optimising the perturbation amplitude in both, constant amplitude and frequency dependant amplitude strategies.
\end{abstract}

Keywords: Electrochemical Impedance Spectroscopy (EIS), Perturbation amplitude, Harmonic analysis, Linearity condition, Frequency dependant amplitude. 


\section{Introduction}

Today, the Electrochemical Impedance Spectroscopy (EIS) technique has become a widespread and well-established technique in the electrochemical field [1]. This technique can be applied in order to study the mechanisms of electrochemical reactions, for measuring the electric and transport properties of materials, for exploring the properties of porous materials, and for studying complex interfaces [2]. The power of this electrochemical technique arises from its ability to distinguish the different physicchemical processes undergoing at different timescales in the system [3]. This property makes it suitable for a wide range of applications such as fuel cells [4-6], batteries [713], electrolyzers [14], corrosion [15-17], coatings [18-19], membranes [20], environmental applications $[21,22]$, electrochemical sensors $[23,24]$ and supercapacitors [25-27].

This technique consists in the measurement of the complex impedance of a system at different perturbation frequencies; whence the name of "spectroscopy" [28]. In order to determine the complex impedance in galvanostatic mode, a sinusoidal current perturbation is applied to the system; and the resulting potential signal is measured. In potentiostatic mode, a potential perturbation is used, and the resulting current signal is measured. Once the response of the system has been obtained, the complex impedance is determined using Ohm's generalized law:

$$
Z(\omega)=\frac{\mathcal{F}[U(t)]}{\mathcal{F}[I(t)]}
$$

Where $Z(\omega)$ denotes the complex impedance of the system at angular frequency $\omega=$ $2 \pi f . U(t)$ and $I(t)$ respectively stand for the potential and the current signals in the time domain. And finally, $\mathcal{F}$ is the Fourier transform operator. The Fourier transform of an arbitrary function in the time domain, $h(t)$, is defined as [29]:

$$
\mathcal{F}[h(t)](\vartheta)=\int_{-\infty}^{+\infty} h(t) \cdot e^{-2 \pi j \vartheta t} \cdot d t
$$

Where $j$ denotes the imaginary unit, $\sqrt{-1}$; and $\vartheta$ represents the frequency domain independent variable.

The impedance concept is a generalization of the DC electric resistance concept to AC perturbed systems. The complex impedance contains two components: the real part $\left(Z^{\prime}\right)$ and the imaginary part $\left(Z^{\prime \prime}\right)$, in rectangular representation; or the modulus $(|Z|)$ and the phase angle $(\varphi)$, in polar representation. On the one hand, the impedance modulus gives the ratio between the amplitude of the voltage signal and the amplitude 
of the current signal; and on the other hand, its argument gives the phase angle of the voltage signal with respect to the current signal [30].

Macdonald and Sikora stated that the validity of Ohm's complex law, and thus of the impedance concept, requires the fulfilment of 4 critical hypotheses: causality, linearity, stability, and finiteness [31]. However, it has been proved that the finiteness condition is not critical; therefore there are only 3 critical hypotheses: causality, linearity and stability [32]. If any of these conditions is violated, the obtained EIS results may be biased; and no reliable conclusions can be extracted from them [33]. For this reason, it is crucial to assess if the 3 critical hypotheses were met during EIS measurements, before performing any further analysis [34].

Linear systems are systems in which the superposition principle holds [35], in other words, systems which response to a sum of perturbations is the sum of the responses of the system to the individual perturbations. A great number of works have studied the effects of the non-fulfilment of the linearity condition: some examples of these works are the works of Darowicki and co-workers [36-39]; the works of Diard and co-workers [40-45]; and the works of Van Gheem's team [46-47]. It has been shown that the main effect of nonlinearity is the generation of non-fundamental harmonics in the output signal [48]. When a sinusoidal perturbation is applied to a linear system, the response signal is a monofrequency sinusoidal signal of same frequency [49]. This being the basis of the impedance definition [30]. On the contrary, when a sinusoidal perturbation is applied to a nonlinear system, the response signal is composed by a fundamental signal and its harmonics [50]. The harmonic generation may distort the measured EIS spectrum; invalidating the impedance definition. Montella and Diard implemented a Wolfram ${ }^{\circledR}$ Demonstration Project [51] that illustrates very clearly the effects of the nonlinearity of a Tafelian system on its EIS spectrum [52].

In EIS context, nonlinear effects, such as nonlinear kinetics (Buttler-Volmer's equation) and saturation, often appear in the investigation of electrochemical systems [53-54]. On account of this reason, low amplitude perturbation must be used for EIS measurements [55], in order to ensure the fulfilment of the linearity condition [56]. However, low perturbation amplitudes lead to low signal-to-noise ratios [57], which affects negatively the quality of the measured EIS spectra. Thus, the selection of the perturbation amplitude must be done on the basis of the trade-off between the linearity condition fulfilment and the maximization of the signal-to-noise ratio [2]: the perturbation amplitude has to be low enough in order to guarantee the fulfilment of the linearity condition [58]; and it has to be high enough in order to achieve an acceptable signal-tonoise ratio [59]. The optimum perturbation amplitude is defined as the perturbation amplitude that maximizes the signal-to-noise ratio without violating the linearity 
condition [60]. This optimum amplitude varies from one system to another; and for a given system, it may even change from one operation point to another.

The optimum amplitude can be determined using a linearity assessment method. A great number of linearity verification techniques can be found in literature [61]. These linearity assessment methods can be classified into 3 main types: 1 . Experimental methods; 2 . Kramers-Kronig based methods; and 3. harmonic analysis based methods [28].

Firstly, the experimental methods include AC plots, resolution plots and Lissajous figures. Linearity can be assessed in real time during EIS measurement using these plots. The aforementioned plots directly monitor the perturbation and response raw signals in the time domain [2]. Nowadays, most if not all EIS measurement commercial softwares include this kind of tools, which can be displayed during data acquisition. This kind of linearity assessment methods has 2 major drawbacks. On the one hand, they are qualitative methods. Therefore, they are able to detect nonlinearities, but cannot quantify the nonlinearity level. On the other hand, while the distortion of AC plots and Lissajous figures is easily recognizable for severe nonlinearities; the distortion of these plots due to low and moderate nonlinearities may be ambiguous, and its identification can be subjected to the subjectivity of the analyst [2]. For this reason, the use of this type of linearity assessment methods should be restricted to auxiliary experimental validation during the measurement; and should be avoided in rigorous perturbation amplitude optimization.

Secondly, the Kramers-Kronig methods are based on the Kramers-Kronig relations, which are integral relations that relate the real and the imaginary parts of complex quantities that satisfy the conditions of causality, linearity, and stability [30]. The power of the Kramers-Kronig relations as a validation tool of EIS spectra has been widely proven in bibliography. Agarwal and Orazem presented in their work [62] an extensive review of the available methods for the Kramers-Kronig relations application for EIS spectra validation. The main examples of Kramers-Kronig based validation methods are the Voight method developed by Boukamp and co-workers [63-64]; and the measurement model method developed by Orazem's group [34, 62, 65-72]. Urquidi-Macdonald and co-workers observed that the Kramers-Kronig relations were highly insensitive to nonlinearity [35]. More recently, Hirschorn and Orazem have shown that KramersKronig relations are only sensitive to nonlinearities if at least part of the spectrum has been measured in a range above the threshold frequency of the system; and even then, the sensitivity is not very high [73]. These observations were conformed in a previous work [74]. The low sensibility to nonlinearities of this kind of validation technique makes it unsuitable for perturbation amplitude optimization. 
Finally, the harmonic analysis based methods assess linearity through the quantification of the non-fundamental harmonic content of the response signal. These methods are based on the analysis of the system's response in the frequency domain. Several examples of this kind of linearity assessment method can be found in literature. The method developed by Popkirov and Schindler [48, 75], the Pintelon's team method [7677], and the total distortion based method developed in a previous work [78] are some examples of the harmonic analysis based linearity assessment methods available in bibliography. The advantages of this kind of linearity assessment methods are that they are quantitative methods, able to quantify the level of nonlinearity in an objective manner; and they have a great sensibility to nonlinearities.

In a previous work, a linearity assessment method based on the harmonic analysis of the response signal was presented and experimentally validated [79]. This work's aim is to apply the aforementioned linearity assessment method in order to determine the optimum perturbation amplitude for EIS measurements of a highly nonlinear electrochemical system: an alkaline hydrogen evolution cell. Two different strategies were considered in this work: on the one hand, the traditional constant amplitude perturbation strategy; and on the other hand, a frequency dependant perturbation amplitude strategy. 


\section{Linearity assessment method}

The linearity assessment method that was used in this work for perturbation amplitude optimization was described in a previous work [79]. The method breaks down in 3 main steps. In the first step, the EIS spectrum is measured as usual. However, instead of saving only the impedance value for each excited frequency; the raw current and voltage signals in the time domain, $I(t)$ and $U(t)$, are also stored for each excited frequency. In a second step, a Fast Fourier Transform (FFT) algorithm is used in order to transform the signals in the time domain into the frequency domain. FFT is a well-known algorithm used to transform a signal in the time domain into the frequency domain. Further details on FFT can be found in any review on spectrum analysis (v.g. the work of Kay and Marple $[80])$. Using this algorithm, the current and voltage signals in the frequency domain, $\hat{I}(\vartheta)$ and $\widehat{U}(\vartheta)$, are obtained for each excited frequency.

Once the signals in the frequency domain have been obtained, in the third step of the linearity assessment method, the level of non-fundamental harmonics in the output signal (potential, in this case) is quantified using the following parameter:

$$
\wp U=\max _{\mathrm{k} \neq 1} 20 \cdot \log _{10}\left(\frac{|\widehat{U}|_{k}}{|\widehat{U}|_{1}}\right)
$$

Where $|\widehat{U}|$ denotes the amplitude of the Fourier transform of the output signal. Subscript 1 refers to the fundamental component of the signal, whereas subscripts greater or equal to 2 refer to non-fundamental harmonics of the signal. This parameter quantifies in decibels $(\mathrm{dB})$ the ratio between the most important non-fundamental harmonic of the signal, and its fundamental component.

After applying this method, the values of parameter $\wp U$ are obtained for each one of the frequencies at which the impedance was measured in the first step. The obtained results can be analysed using two tools: the $\wp U$ curves and the critical parameter. On the one hand, $\wp U$ curves correspond with the representation of $\wp U$ parameter versus the excited frequency. These curves allow evaluating individually the linearity of each frequency. As it was observed in a previous work [79], these curves are very useful for identifying which frequencies are more susceptible to present nonlinearities in the studied system. On the other hand, the critical frequency is defined as the excited frequency with the highest harmonic content; and the critical parameter, $\wp U_{c}$, is defined as the value of $\wp U$ parameter at the critical frequency. An analog definition stablishes that:

$$
\wp U_{\mathrm{c}}=\max _{\mathrm{i} \in\left\{1 ; 2 ; \cdots ; N_{f}\right\}} \wp U_{\mathrm{i}}
$$


Where $N_{f}$ denotes the number of frequencies at which the impedance was measured. This critical parameter provide an overall assessment of linearity by considering the most unfavorable frequency: the frequency of the EIS spectrum with the highest harmonic content.

Figure 1 of [79] shows a detailed outline of the linearity assessment method used in this work. As explained in the aforementioned work, the linearity assessment method was implemented in Labview $\Re$ : the developed program takes as input the output of Nova which contains the digitalized raw signals (current and voltage) in the time domain; and the transformed signals in the frequency domain. The program reads the later, and calculates parameter $\wp U$. Repeating this process for each one of the excited frequencies, the value of $\wp U$ is obtained for every frequency; obtaining the corresponding $\wp U$ curve. Then, the critical parameter is calculated. 


\section{Methodology}

The aim of this work is to obtain the optimum perturbation amplitude for EIS measurements of a given system: the cathodic electrode of an alkaline water electrolyser. The common approach used in literature to tackle this problem is to study the effect of the perturbation amplitude on the impedance spectra: in this approach, the biggest perturbation amplitude that does not affect significantly the measured spectrum is selected. For example, this strategy was used by Yuan and co-workers [81]. However, this method has several weaknesses. Firstly, the electrochemical impedance spectrum may vary because of other causes than the perturbation amplitude (i.e. non stationarity). Consequently, a suboptimal amplitude may be selected because a variation in the impedance spectrum was observed, but that variation was not due to the perturbation amplitude. Secondly, "affect significantly" is quite fuzzy: the criterion to distinguish a significant distortion due to the perturbation amplitude from the inherent variability of the measurement is quite unclear, and it is generally left to the subjectivity of the annalist. Finally, this kind of approach is only useful for a constant amplitude (frequency independent) strategy; but it cannot be used for a frequency dependent amplitude strategy, in which a different amplitude is selected for each frequency. The linearity assessment method overcomes all these limitations.

In this work, two strategies were considered: a constant amplitude strategy and a frequency dependent amplitude strategy. In order to achieve this work's goal, the galvanostatic impedance spectra of the studied system were measured using different perturbation amplitudes. The linearity assessment method, described in the previous section, was applied to each one of the obtained spectra: the $\wp U$ curve and the critical parameter, $\wp U_{c}$, were obtained for each perturbation amplitude. On the one hand, the critical parameters were used for the constant amplitude strategy, while the $\wp U$ curves for individual frequencies were used for the frequency dependent amplitude strategy.

In the constant amplitude strategy case, the critical parameter curve was used in order to obtain the optimum perturbation amplitude. The critical parameter curve consists in the representation of the critical parameter $\wp U_{c}$ versus the perturbation amplitude. As it was shown in [79], the optimum amplitude in the constant amplitude strategy case is determined by the minimum of the critical parameter curve.

In the frequency dependent amplitude strategy case, the $\wp U$ curves for individual frequencies were used in order to obtain the optimum perturbation amplitude for each frequency. These curves correspond with the representation of the $\wp U$ parameter for a given frequency versus the perturbation amplitude. Of course, there is one such curve for each excited frequency. The optimum amplitude for each frequency is given by the minimum of the $\wp U$ curve for that frequency. 


\section{Experimental work}

The experimental setup is shown in figure 1. The system corresponds with the cathodic electrode of an alkaline water electrolyser. A detailed description of the system is presented in the works of Herraiz-Cardona and co-workers [82-85]. In these works, it was shown that the system is strongly nonlinear.

The main element of this experimental setup is an electrochemical cell patented by the Dpto. Ingenieria Quimica y Nuclear of Universitat Politècnica de València (Spain) [86]. It consists in a three-electrode electrochemical cell coupled with a heating water circuit that allows controlling the temperature of the cell. One of the electrodes developed by Herraiz-Cardona and co-workers was used as the working electrode. The working electrode was placed in one of the two horizontal positions available in the electrochemical cell. It consists in a nickel electrode produced at very high current densities using the procedure described in [85]. The geometric area of the electrode used in this work was of $0.5 \mathrm{~cm}^{2}$, and its rugosity factor was around 1000 . This leads to an active surface of around $500 \mathrm{~cm}^{2}$. The counter-electrode, a nickel foam with very high surface area (Incofoam $® 0.17 \mathrm{~cm}$ thick and 50 pores per linear inch), was placed in one of the three vertical openings available in the electrochemical cell. The mentioned counter-electrode was $1 \mathrm{~cm}$ wide, and its submerged length was of $5 \mathrm{~cm}$. Its active area was around 2 orders of magnitude higher than the active area of the working electrode. Finally, a commercial $\mathrm{Ag} / \mathrm{AgCl}(3 \mathrm{M} \mathrm{KCl})$ electrode was used as the reference electrode, which was placed in another of the three vertical openings of the electrochemical cell. The third vertical opening was left open to the atmosphere, in order to act as a gas vent; and thus, to prevent overpressures inside the electrochemical cell due to the gases produced during the water electrolysis. An oxygen free $30 \mathrm{wt} . \% \mathrm{KOH}$ solution was used as electrolyte. Before each experimental session, fresh electrolyte was prepared using 85 wt.\% $\mathrm{KOH}$ Panreac ${ }^{\circledR}$ lentils. To avoid the carbonation of the electrolytic solution, which would cause an increase of the electrolyte resistance over time, the electrolyte was deaerated by bubbling nitrogen for 15 minutes just before starting the experiments.

As explained in the methodology section, in order to obtain the optimum perturbation amplitude, the EIS spectrum of the system was measured at different perturbation amplitudes. In this case, 12 different perturbation amplitudes were considered: $0.1 \mathrm{~mA}$; $0.5 \mathrm{~mA} ; 1 \mathrm{~mA} ; 2 \mathrm{~mA} ; . . . ; 10 \mathrm{~mA}$ (peak to peak amplitudes). All the experiments were carried out at $30^{\circ} \mathrm{C}$, in galvanostatic mode. In preliminary works, it was observed that the most nonlinear operation point of the system is obtained for an operation DC current of $-10 \mathrm{~mA}$, which corresponds to an overvoltage of around $-200 \mathrm{mV}$. In these conditions, the production of bubbles is low, and therefore the bubble evolution effect is negligible. The minus sign indicates that the working electrode acts as the cathode of the system. All the experiments were performed at this DC current. The maximum 
perturbation amplitude of $10 \mathrm{~mA}$ was selected in order to guarantee that the working electrode acts as the cathode of the cell during the whole EIS measurement, even for the maximum perturbation amplitude.

The EIS measurements were performed using an Autolab® $302 \mathrm{~N}$ potentiostat/galvanostat with FRA module, controlled using NOVA® software. The measurement frequency range was $10 \mathrm{kHz}-5 \mathrm{mHz}$, with 10 frequencies per decade. Table 1 lists the measurement parameters used for all the measurements. The meaning of each one of these measurement parameters was widely explained in a previous work [87]. In the mentioned work, a methodology for the optimization of the measurement parameters is presented.

Measurements were obtained in triplicate in order to control the reproducibility of the obtained results. Replicates of each measurement were not performed sequentially; instead, the experiments were done in three different blocks as shown in table 2 , which gives the sequence order in which the experiments were performed. As it can be seen in the aforementioned table, the order of the experiments inside each block was randomized; instead of performing the experiments in order of increasing or decreasing amplitude. The random order strategy was selected since randomization makes the factors of time and amplitude independent: this means that it allows to distinguish the effects of the perturbation amplitude from the effects of possible time drifts. On the contrary, if an increasing amplitude strategy is used, it is not possible to know if the observed trends are due to a time drift of the system or to the effect of the perturbation amplitude.

Before each experiment, a pre-treatment was applied to the working electrode in order to ensure similar surface conditions in all experiments. The applied pre-treatment consisted in applying a $-1.6 \mathrm{~V}$ vs. $\mathrm{Ag} / \mathrm{AgCl}$ potential (overvoltage of around $-350 \mathrm{mV}$ ) during 30 minutes. This treatment was done in order to reduce any oxide film that could exist on the surface of the porous electrode [84], in order to guarantee that the surface of the electrode was in similar conditions in all the experiments. This pre-treatment is critical in order to ensure the reproducibility of the results from one experiment to another. 


\section{$\underline{\text { 5. Experimental results }}$}

\subsection{Constant amplitude strategy}

Figure 2 presents the critical parameter curve of the output signal. In this curve the critical parameter, $\wp U_{c}$, is represented versus the perturbation amplitude. Next to each point, the critical frequency for that perturbation amplitude is displayed. Two different trends can be identified in figure 2. On the one hand, for low perturbation amplitudes, an increase in the amplitude causes a drop of the critical parameter of the output signal. On the other hand, for high perturbation amplitudes, an increase in the perturbation amplitude causes an increase of the critical parameter. The emergence of the two trends is due to the fact that an increase in the perturbation amplitude causes two antagonist effects, each one dominating in one of the two identified perturbation amplitude ranges. The first effect of an increase of the perturbation amplitude is the improvement of the signal-to-noise ratio. The second effect is the generation of higher levels of nonfundamental harmonics due to nonlinear effects. For low amplitudes, the first effect dominates over the second one. For this reason, in this perturbation amplitude range, the net effect of an increase of the perturbation amplitude is a drop in the critical parameter. In this zone, the system behaves linearly-like; and thus, this zone is defined as the linear behaviour zone of the system, identified on figure 2. On the contrary, for high perturbation amplitudes, the second effect dominates over the first one. The result is that the net effect of an increase of the perturbation amplitude, in this amplitude range, is an increase of the critical parameter. Therefore, the nonlinear behaviour of the system is significant for perturbation amplitudes in this range. For this reason, as identified on figure 2 , this amplitude range is defined as the nonlinear behaviour zone of the system.

In addition, in figure 2 it can be observed that the critical frequency changes changes from one zone to the other. In the linear behaviour zone, the critical frequency is $25 \mathrm{~Hz}$ for all the perturbation amplitudes. This frequency is the perturbation frequency that presents a higher noise level, due to the coupling with the electric grid. This is consistent with the linear behaviour zone definition: in this zone (where noise is dominant over nonlinear effects), the critical frequency corresponds with the frequency with a higher noise level. In the nonlinear behaviour zone, the critical frequency is the lowest frequency of the frequency range $(5 \mathrm{mHz})$ for every perturbation amplitude. This is due to the fact that nonlinear effects only appear at low frequencies [78]. Therefore, in the nonlinear behaviour zone (where nonlinear effects are dominant over noise) the critical frequency is the minimum measured frequency, which is the frequency that exhibits higher nonlinear effects. Consequently, the critical frequency shift is an indicator of the domination switch from noise to nonlinear effects. This shift delimits the linear and the nonlinear zones. 
The minimum point of the critical parameter curve identifies the perturbation amplitude that maximizes the signal-to-noise ratio, while maintaining the nonlinear effects in a negligible level. This minimum point delimits the linear behaviour zone of the system, and its nonlinear behaviour zone. By the definition of optimum perturbation amplitude presented in the introduction section, the optimum perturbation is given by the horizontal coordinate of the minimum point. Consequently, it can be deduced from figure 2 that the optimum amplitude for performing EIS measurements of the considered system with a constant amplitude strategy, lays in the range $1 \mathrm{~mA}$ to $3 \mathrm{~mA}$. However, with the available data, it can be deduced that the best perturbation of the list of applied perturbations corresponds with the $2 \mathrm{~mA}$ perturbation.

\subsection{Frequency dependent amplitude strategy}

Figure 3 shows the $\wp U$ curves obtained for 2 different perturbation amplitudes ( $2 \mathrm{~mA}$ and $10 \mathrm{~mA}$ ). These curves consist in the representation of the $\wp U$ parameter versus the perturbation signal frequency, for a given perturbation amplitude. These curves display the characteristic shape described in detail in a previous work [79]. Comparing both $\wp U$ curves, two different trends can be identified: for low frequencies, an increase in the perturbation amplitude causes the $\wp U$ curve to shift to higher values; while for high frequencies, an increase in the perturbation amplitude causes the $\wp U$ curve to shift to lower values. It can be deduced that, in this perturbation amplitude range, the system behaves quasi-linearly for high frequencies; and nonlinearly for low frequencies. This is due to the existence of a frequency threshold above which nonlinear effects are not generated even for very high perturbation amplitudes $[56,73]$. This shows that the sensibility of a system to nonlinear effects is frequency dependent: for a given perturbation amplitude, a given system can behave linearly in a frequency zone and nonlinearly in another frequency zone. This observation motivates the need to consider a frequency-dependent perturbation amplitude: large perturbation amplitudes may be applied in the linear frequency zone (in order to improve as much as possible the signalto-noise ratio); and low perturbation amplitudes have to be applied in the nonlinear frequency zone (in order to avoid distortions of the spectrum due to nonlinear effects).

Figure 4 displays the individual frequency $\wp U$ curves of 4 different excitation frequencies $(10 \mathrm{kHz}, 50 \mathrm{~Hz}, 20 \mathrm{~Hz}$, and $5 \mathrm{mHz}$ ). These curves correspond with the representation for a given frequency of the $\wp U$ parameter versus the perturbation amplitude. On the one hand, for high frequencies $(10 \mathrm{kHz}$ and $50 \mathrm{~Hz})$, only one trend is observed: $\wp U$ decreases (logarithmically) with the perturbation amplitude. This is consistent with the expression of $\wp U(f)$ obtained for the linear behaviour zone in [79]. It can be deduced that the system behaves linearly for every perturbation amplitude for these frequencies. Consequently, the maximum perturbation amplitude (10 $\mathrm{mA}$ ) can be used for these frequencies in order to maximize the signal-to-noise ratio, since no 
nonlinear effects appear at these frequencies. On the other hand, for low frequencies $(20 \mathrm{~Hz}$ and $5 \mathrm{mHz}$ ) two trends can be observed. The explanation of the two trends was already presented in the previous section. The optimum perturbation amplitude for these frequencies is determined from the minimum of the curve.

Using this strategy for every excitation frequency, the optimum amplitude was obtained for each frequency. The results are shown in figure 5 . It can be observed that high perturbation amplitudes should be used for high frequencies; while low perturbation amplitudes should be used for low frequencies. This is consistent with the fact that nonlinear effects only appear under a threshold frequency. Above this frequency, very high amplitudes can be used (maximization of signal-to-noise ratio) without generating significant nonlinear effects. 3 peaks can be observed in the aforementioned figure: the maximum amplitude should be used for the $50 \mathrm{~Hz}$, the $25 \mathrm{~Hz}$ and the $12.5 \mathrm{~Hz}$ points. These points are below the frequency threshold, since nonlinear effects start appearing at $250 \mathrm{~Hz}$. However, these points present very high noise levels because of coupling with the electric grid. That is the reason why at these frequencies nonlinear effects are masked by noise, even at the maximum perturbation amplitude. 


\section{$\underline{6 . \text { Conclusions }}$}

In conclusion, the presented linearity method was successfully used to obtain the optimum perturbation amplitude for EIS measurements of the cathodic electrode of an alkaline water electrolyser for two strategies: a frequency independent perturbation amplitude strategy and a frequency dependent perturbation amplitude strategy.

For the frequency independent perturbation amplitude strategy the optimum amplitude is $2 \mathrm{~mA}$; whereas for the frequency dependent perturbation amplitude strategy, high amplitude should be used for high frequencies and low amplitude for low frequencies.

Even if in this work the method has only been applied to this particular system; the method can be used for perturbation amplitude optimization in any system. The great advantage of this method is that it allows a rigorous and quantitative perturbation amplitude selection. Furthermore, the presented method allows to obtain the optimum perturbation amplitude for each frequency in a frequency dependent strategy. This kind of strategy is required in systems in which frequency has an important effect on the sensibility of a system to nonlinear effects. 


\section{Nomenclature}

\section{Latin letters}

$\mathcal{F} \quad$ Fourier transform operator

$f \quad$ Frequency $(\mathrm{Hz})$

$f_{c} \quad$ Critical frequency $(\mathrm{Hz})$

I Current in the time domain $(A)$

$\hat{I} \quad$ Current in the frequency domain $(A)$

$N_{f} \quad$ Number of measured frequencies

j Imaginary unit

$t \quad$ Time domain independent variable $(s)$

$U \quad$ Voltage in the time domain $(V)$

$\widehat{U} \quad$ Voltage in the frequency domain $(V)$

$Z \quad$ Complex impedance $(\Omega)$

$Z^{\prime} \quad$ Impedance real part $(\Omega)$

$Z^{\prime \prime} \quad$ Impedance imaginary part $(\Omega)$

\section{Greek letters}

$\Delta I \quad$ Galvanostatic perturbation amplitude $(A)$

$\vartheta \quad$ Frequency domain independent variable $(\mathrm{Hz})$

$\omega \quad$ Angular frequency $\left(\mathrm{rad} \cdot \mathrm{s}^{-1}\right)$

$\wp U \quad$ Ratio between the most important non-fundamental harmonic of the voltage signal, and its fundamental component $(d B)$

$\wp U_{\mathrm{c}} \quad$ Critical parameter for the voltage signal $(d B)$ 


\section{Acknowledgments}

The authors are very grateful to the Generalitat Valenciana for its economic support in form of Vali+d grant (Ref: ACIF-2013-268). 


\section{Bibliography}

[1] D.D. Macdonald, Electrochim. Acta, 51, 1376 (2006).

[2] M.E. Orazem and B. Tribollet, Electrochemical Impedance Spectroscopy, John Wiley \& Sons, New Jersey (2008).

[3] J.J. Giner-Sanz, E.M. Ortega and V. Pérez-Herranz, Int. J. Hydrogen Energ., 40, 11279 (2015).

[4] V. Cascos, A. Aguadero, G. Harrington, M. T. Fernández-Díaz and J. A. Alonso, J. Electrochem. Soc., 164, F3019 (2017).

[5] S. Pang et al., J. Electrochem. Soc., 164, F775 (2017).

[6] R. Kiebach et al., J. Electrochem. Soc., 164, F748 (2017).

[7] J. Chen et al., J. Electrochem. Soc., 164, A1526 (2017).

[8] C. Hwang, K. Lee, H.D. Um, Y. Lee, K. Seo and H.K. Song, J. Electrochem. Soc., 164, A1564 (2017).

[9] Y. Zhang et al., J. Electrochem. Soc., 164, A1695 (2017).

[10] S. Malifarge, B. Delobel and C. Delacourt, J. Electrochem. Soc., 164, E3329 (2017).

[11] A.R. Paulraj, Y. Kiros, B. Skårman and H. Vidarsson, J. Electrochem. Soc., 164, A1665 (2017).

[12] M. Stein, A. Mistry and P.P. Mukherjee, J. Electrochem. Soc., 164, A1616 (2017).

[13] M.D. Murbach and D.T. Schwartz, J. Electrochem. Soc., 164, E3311 (2017).

[14] J.J. Giner-Sanz, E.M. Ortega and V. Pérez-Herranz, Fuel Cells doi 10.1002/fuce.201600137 (2017).

[15] J. Katić, M. Metikoš-Huković, I. Šarić and M. Petravić, J. Electrochem. Soc., 164, C383 (2017).

[16] J. Yang, Y. Yang, A. Balaskas and M. Curioni, J. Electrochem. Soc., 164, C376 (2017). 
[17] Y. Takabatake, Y. Kitagawa, T. Nakanishi, Y. Hasegawa and K. Fushimi, J. Electrochem. Soc., 164, C349 (2017).

[18] J. Qi, L. Gao, Y. Li, Z. Wang, G. E. Thompson and P. Skeldon, J. Electrochem. Soc., 164, C390 (2017).

[19] Q. Zhang et al., J. Electrochem. Soc., 164, A1503 (2017).

[20] A.A. Moya, J. Phys. Chem. C, 120, 6543 (2016).

[21] D.A. García-Osorio, R. Jaimes, J. Vazquez-Arenas, R.H. Lara and J. Alvarez-Ramirez, J. Electrochem. Soc., 164, E3321 (2017).

[22] Q. Wei et al., J. Electrochem. Soc., 164, H515 (2017).

[23] S. Machado et al., J. Electrochem. Soc., 164, B314 (2017).

[24] P. Balasubramanian, B. Thirumalraj, S.M. Chen and P. Barathi, J. Electrochem. Soc., 164, H503 (2017).

[25] J. Jiang, J. Electrochem. Soc., 164, H5043 (2017).

[26] K.Y. Wang, Y.K. Chiu and H.C. Cheng, J. Electrochem. Soc., 164, A1587 (2017).

[27] C. Chang, X. Yang, S. Xiang, X. Lin, H. Que and M. Li, J. Electrochem. Soc., 164, A1601 (2017).

[28] A. Lasia, Electrochemical Impedance Spectrscopy and its applications, Springer, London (2014).

[29] M.G. Gray and J.W. Goodman, Electrochemical Impedance Spectrscopy and its applications, Springer, New York (1995).

[30] E. Barsoukov and J.R. Macdonald, Impedance Spectroscopy. Theory, experiment and applications, John Wiley \& Sons, New Jersey (2005).

[31] D.D. Macdonald and E. Sikora, Electrochim. Acta, 43, 87 (1997).

[32] M. Schönleber, D. Klotz and E. Ivers-Tiffée, Electrochim. Acta, 131, 20 (2014).

[33] J.E. Garland, C.M. Pettit and D. Roy, Electrochim. Acta, 49, 2623 (2004). 
[34] M.E. Orazem and B. Tribollet, Electrochim. Acta, 53, 7360 (2008).

[35] M. Urquidi-Macdonald, S. Real and D.D. Macdonald, Electrochim. Acta, 35, 1559 (1990).

[36] K. Darowicki, J. Electroanal. Chem., 394, 81 (1995).

[37] K. Darowicki, Electrochim. Acta, 40, 439 (1995).

[38] K. Darowicki, Electrochim. Acta, 42, 1781 (1997).

[39] J. Smulko and K. Darowicki, J. Electroanal. Chem., 545, 59 (2003).

[40] J.P. Diard, B. Le Gorrec and C. Montella, Electrochim. Acta, 39, 539 (1994).

[41] J.P. Diard, B. Le Gorrec and C. Montella, J. Electroanal. Chem., 377, 61 (1994).

[42] J.P. Diard, B. Le Gorrec and C. Montella, J. Electroanal. Chem., 432, 27 (1997).

[43] J.P. Diard, B. Le Gorrec and C. Montella, J. Electroanal. Chem., 432, 41 (1997).

[44] J.P. Diard, B. Le Gorrec and C. Montella, J. Electroanal. Chem., 432, 53 (1997).

[45] J.P. Diard, B. Le Gorrec and C. Montella, Electrochim. Acta, 42, 1503 (1997).

[46] E. Van Gheem et al., Electrochim. Acta, 49, 4753 (2004).

[47] E. Van Gheem et al., Electrochim. Acta, 51, 1443 (2006).

[48] G.S. Popkirov and R.N. Schindler, Electrochim. Acta, 40, 2511 (1995).

[49] M. Kiel, O. Bohlen and D.U. Sauer, Electrochim. Acta, 53, 7367 (2008).

[50] W. Lai, Electrochim. Acta, 55, 5511 (2010).

[51] C. Montella and J.P. Diard, Nonlinear Impedance of Tafelian Electrochemical Systems, Wolfram Demonstrations Project, 2014, http://demonstrations.wolfram.com/ NonlinearImpedanceOfTafelianElectrochemicalSystems/.

[52] C. Montella, J. Electroanal. Chem, 672, 17 (2012).

[53] J.J. Giner-Sanz, E.M. Ortega and V. Pérez-Herranz, Fuel Cells, 15, 479 (2015). 
[54] J.J. Giner-Sanz, E.M. Ortega and V. Pérez-Herranz, Int. J. Hydrogen Energ., 39, 13206 (2014).

[55] N.S. Kaisare et al., Electrochim. Acta, 56, 7467 (2011).

[56] B. Hirschorn, B. Tribollet and M.E. Orazem, Israel J. Chem., 48, 133 (2008).

[57] S.N. Victoria and S. Ramanathan, Electrochim. Acta, 56, 2606 (2011).

[58] C. Hernandez-Jaimes et al., Chem. Eng. Sci., 137, 1 (2015).

[59] X.Z. Yuan et al., Electrochemical impedance spectroscopy in PEM fuel cells. Fundamentals and applications, Springer, London (2010).

[60] J.J. Giner-Sanz, E.M. Ortega and V. Pérez-Herranz, Fuel Cells, 16, 469 (2016).

[61] F. Fasmin and R. Srinivasan, J. Solid State Electr., 19, 1833 (2015).

[62] P. Agarwal, M.E Orazem and L.H. Garcia-Rubio, J. Electrochem. Soc., 142, 4159 (1995).

[63] B.A. Boukamp and J.R. Macdonald, Solid State lonics, 174, 85 (1994).

[64] B.A. Boukamp, J. Electrochem. Soc., 142, 1885 (1995).

[65] P. Agarwal, M.E. Orazem and L.H. Garcia-Rubio, J. Electrochem. Soc., 139, 1917 (1992).

[66] P. Agarwal et al., J. Electrochem. Soc., 142, 4149 (1995).

[67] P. Agarwal, M.E. Orazem and L.H. Garcia-Rubio, Electrochim. Acta, 41, 1017 (1996).

[68] M.E. Orazem, J.M. Esteban and O.C. Moghissi, Corrosion, 47, 248 (1991).

[69] M.E. Orazem et al., J. Electrochem. Soc., 143, 948 (1996).

[70] M.E. Orazem, P. Shukla and M.A. Membrino, Electrochim. Acta, 47, 2027 (2002).

[71] M.E. Orazem, J. Electroanal. Chemi., 572, 317 (2004). 
[72] P.K. Shukla, M.E. Orazem and O.D. Crisalle, Electrochim. Acta, 49, 2881 (2004).

[73] B. Hirschorn and M.E. Orazem, J. Electrochem. Soc., 156, 345 (2009).

[74] J.J. Giner-Sanz, E.M. Ortega and V. Pérez-Herranz, Electrochim. Acta, 209, 254 (2016).

[75] G.S. Popkirov and R.N. Schindler, Rev. Sci. Instrum., 64, 3111 (1993).

[76] R. Pintelon, E. Louarroudi and J. Lataire, IEEE T. Instrum. Meas., 62, 3361 (2003).

[77] R. Pintelon, E. Louarroudi and J. Lataire, Automatica, 51, 308 (2015).

[78] J.J. Giner-Sanz, E.M. Ortega and V. Pérez-Herranz, Electrochim. Acta, 186, 598 (2015).

[79] J.J. Giner-Sanz, E.M. Ortega and V. Pérez-Herranz, Electrochim. Acta, 211, 1076 (2016).

[80] S.M. Kay and S.L. Marple, Proceedings of the IEEE, 69, 1380 (1981).

[81] X. Yuan et al., J. Power Sources, 161, 920 (2006).

[82] I. Herraiz-Cardona et al., Int. J. Hydrogen Energ., 36, 11578 (2011).

[83] I. Herraiz-Cardona, E.M. Ortega and V. Pérez-Herranz, Electrochim. Acta, 56, 1308 (2011).

[84] I. Herraiz-Cardona, E.M. Ortega and V. Pérez-Herranz, Int. J. Hydrogen Energ., 36, 9428 (2011).

[85] I. Herraiz-Cardona, Desarrollo de nuevos materiales de electrodo para la obtención de hidrógeno a partir de la electrolisis alcalina del agua, PhD Tesis, Universitat Politècnica de València, Valencia, 2012.

[86] J. Garcia-Antón et al. Horizontal cell for electro-optical analysis of electrochemical processes, ES patent P-2000002526, October 2000.

[87] J.J. Giner-Sanz, E.M. Ortega and V. Pérez-Herranz, Electrochim. Acta, 174, 1290 (2015). 


\section{LIST OF TABLES.}

Table 1. EIS measurement parameters

Table 2. Experiment order 


\section{LIST OF FIGURES.}

Figure 1. Experimental setup

Figure 2. Output signal critical parameter curve

Figure 3. $\wp \boldsymbol{U}$ curves for two perturbation amplitudes

Figure 4. $\wp \mathbf{U}$ curve versus perturbation amplitude for different excitation frequencies

Figure 5. Optimum perturbation amplitude for each frequency 
Table 1. EIS measurement parameters

\begin{tabular}{|c|c|}
\hline Measurement parameter & Value \\
\hline Integration time & $1.0 \mathrm{~s}$ \\
\hline Number of integration cycles & $1 \mathrm{cycle}$ \\
\hline Number of stabilization cycles & $10 \mathrm{cycles}$ \\
\hline Maximum stabilization time & $3.0 \mathrm{~s}$ \\
\hline Minimum stabilization cycle fraction & 0.00 \\
\hline
\end{tabular}


Table 2. Experiment order

\begin{tabular}{|c|c|c|c|c|c|c|c|c|}
\hline Order & Block & Experiment & Order & Block & Experiment & Order & Block & Experiment \\
\hline 1 & \multirow{12}{*}{1} & $0.1 \mathrm{~mA}$ & 13 & \multirow{12}{*}{2} & $3 \mathrm{~mA}$ & 25 & \multirow{12}{*}{3} & $9 \mathrm{~mA}$ \\
\hline 2 & & $5 \mathrm{~mA}$ & 14 & & $9 \mathrm{~mA}$ & 26 & & $7 \mathrm{~mA}$ \\
\hline 3 & & $4 \mathrm{~mA}$ & 15 & & $2 \mathrm{~mA}$ & 27 & & $4 \mathrm{~mA}$ \\
\hline 4 & & $1 \mathrm{~mA}$ & 16 & & $0.5 \mathrm{~mA}$ & 28 & & $2 \mathrm{~mA}$ \\
\hline 5 & & $0.5 \mathrm{~mA}$ & 17 & & $5 \mathrm{~mA}$ & 29 & & $0.1 \mathrm{~mA}$ \\
\hline 6 & & $7 \mathrm{~mA}$ & 18 & & $10 \mathrm{~mA}$ & 30 & & $6 \mathrm{~mA}$ \\
\hline 7 & & $3 \mathrm{~mA}$ & 19 & & $7 \mathrm{~mA}$ & 31 & & $0.5 \mathrm{~mA}$ \\
\hline 8 & & $2 \mathrm{~mA}$ & 20 & & $8 \mathrm{~mA}$ & 32 & & $1 \mathrm{~mA}$ \\
\hline 9 & & $6 \mathrm{~mA}$ & 21 & & $0.1 \mathrm{~mA}$ & 33 & & $10 \mathrm{~mA}$ \\
\hline 10 & & $8 \mathrm{~mA}$ & 22 & & $4 \mathrm{~mA}$ & 34 & & $8 \mathrm{~mA}$ \\
\hline 11 & & $9 \mathrm{~mA}$ & 23 & & $1 \mathrm{~mA}$ & 35 & & $5 \mathrm{~mA}$ \\
\hline 12 & & $10 \mathrm{~mA}$ & 24 & & $6 \mathrm{~mA}$ & 36 & & $3 \mathrm{~mA}$ \\
\hline
\end{tabular}




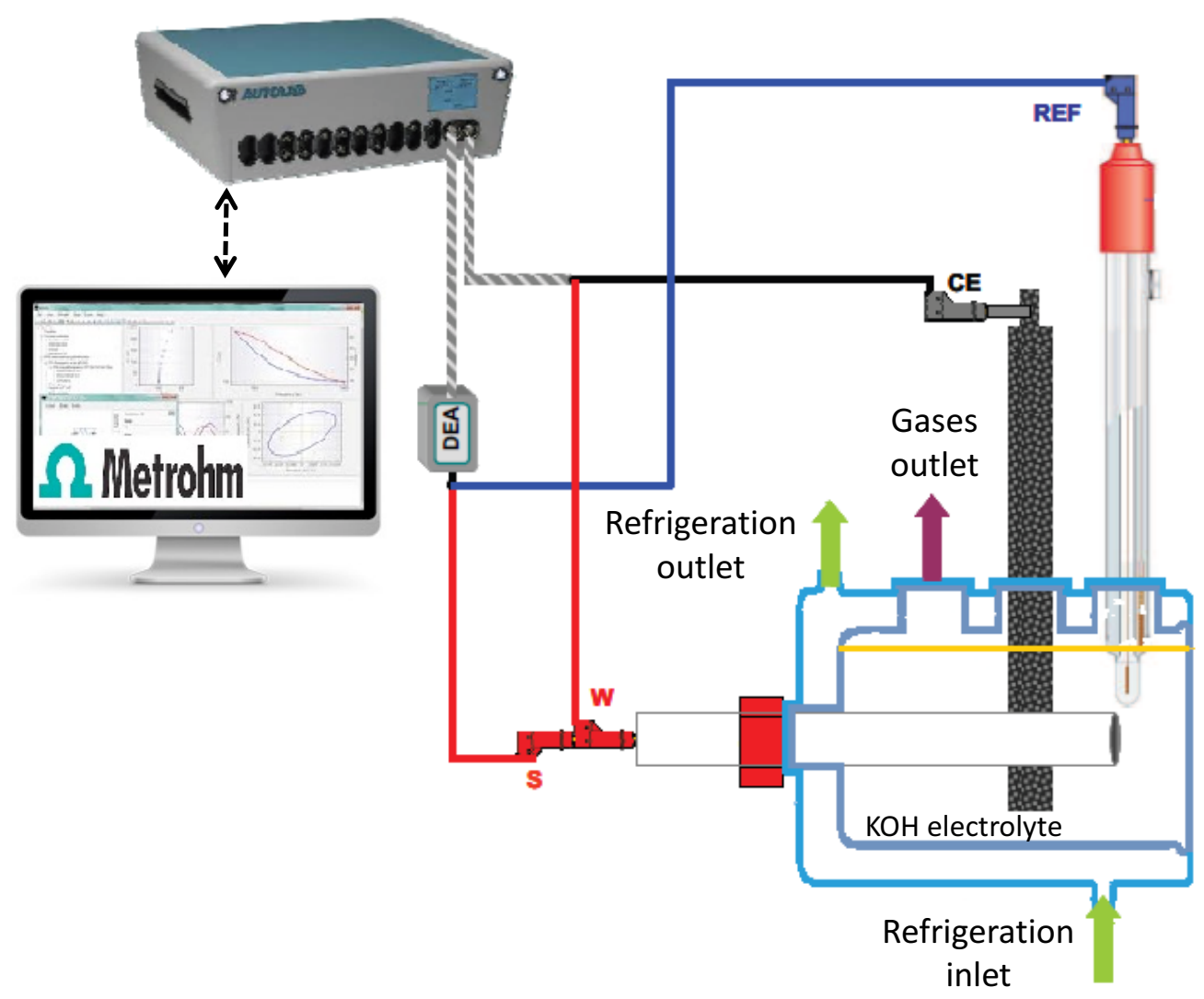

Figure 1. Experimental setup 


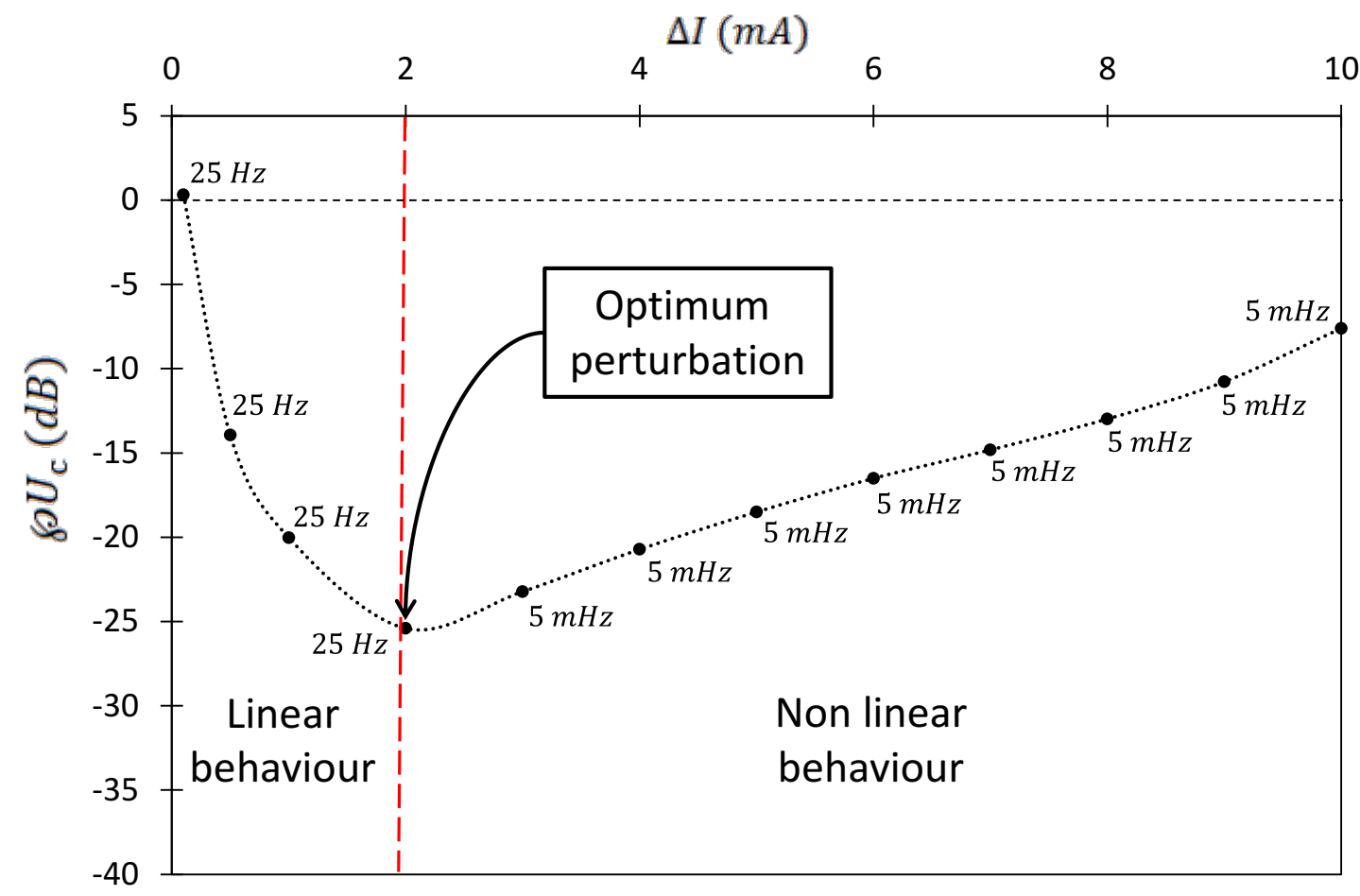

Figure 2. Output signal critical parameter curve 


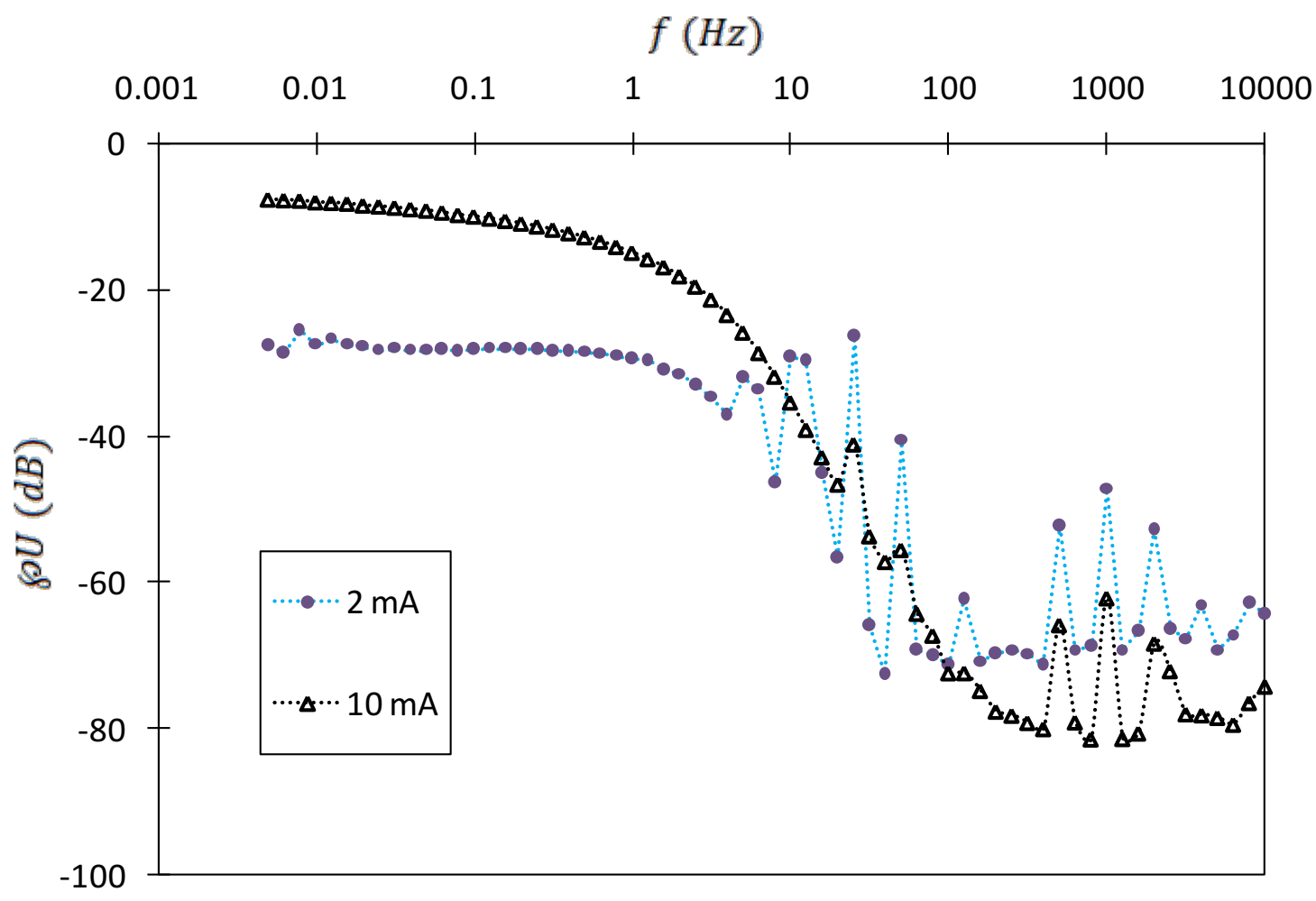

Figure 3. $\wp U$ curves for two perturbation amplitudes 

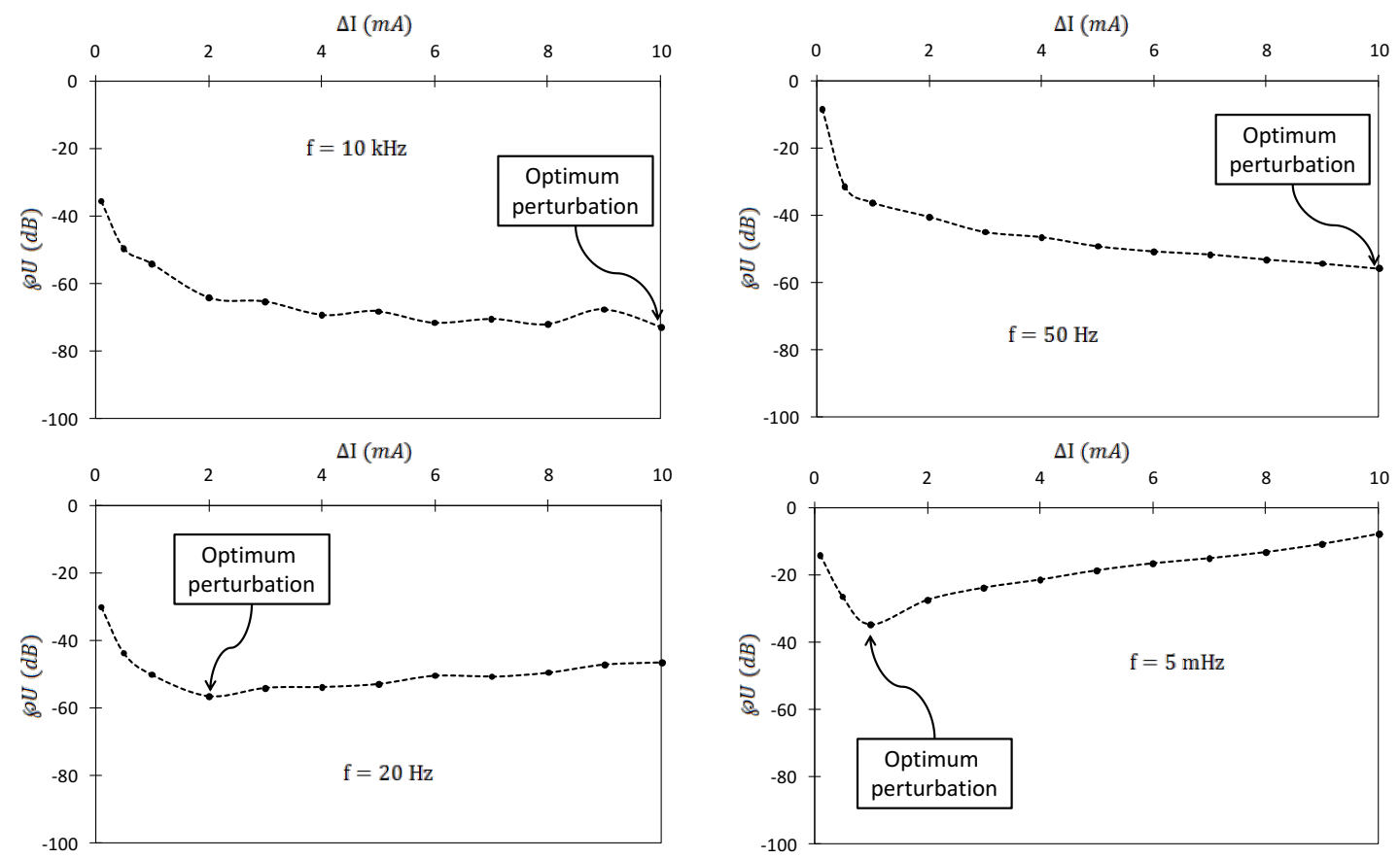

Figure 4. $\wp \mathrm{U}$ curve versus perturbation amplitude for different excitation frequencies 


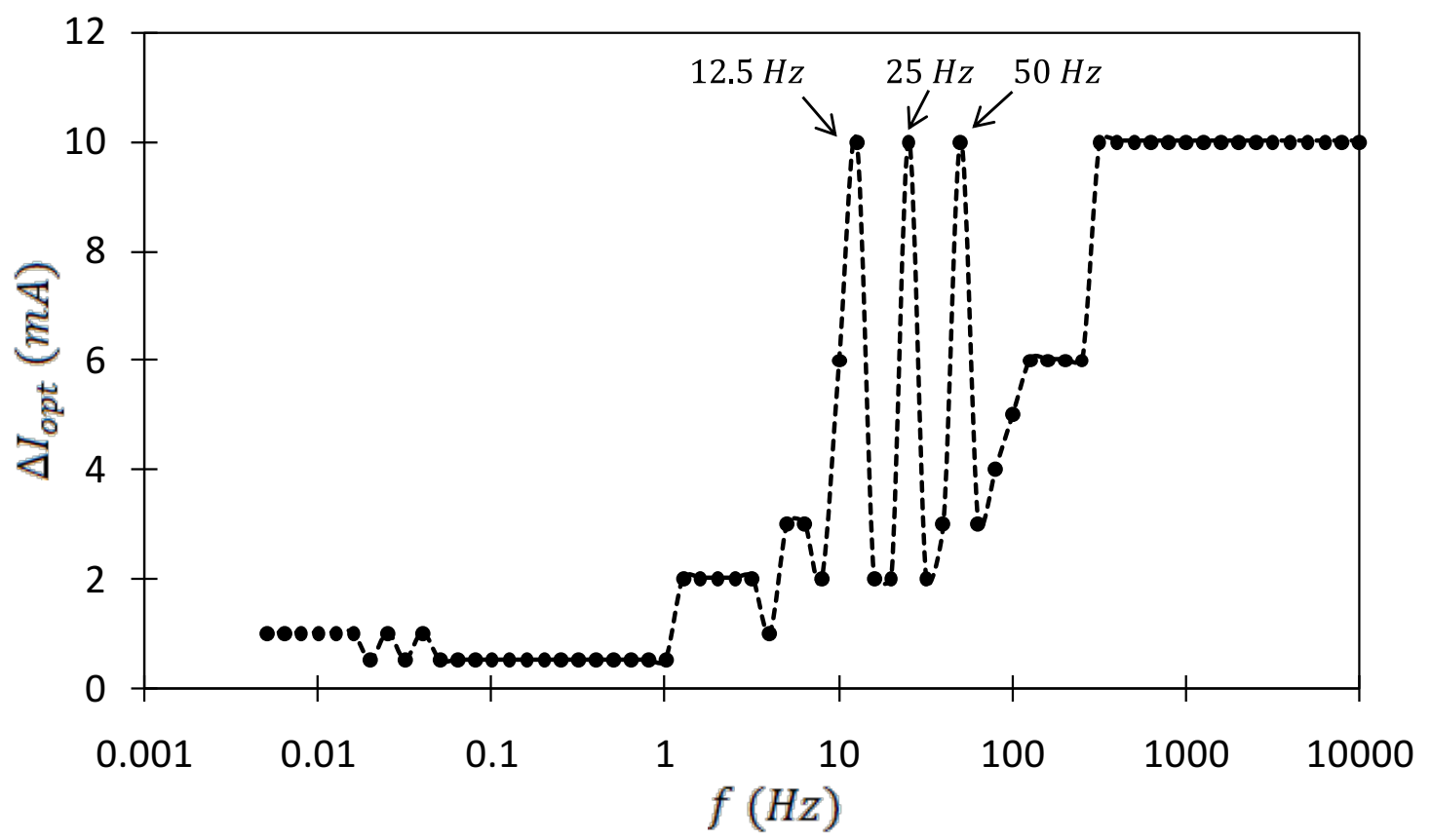

Figure 5. Optimum perturbation amplitude for each frequency 